\title{
Coordinated Observations of SgrA*
}

\author{
Andreas Eckart $^{1,2 *}$ M. García-Marín ${ }^{1}$, G. Witzel ${ }^{1}$, S. König ${ }^{1}$, D. Kunneriath ${ }^{1,2}$, C. \\ Straubmeier ${ }^{1}$, M. Zamaninasab ${ }^{1,2}$ \\ 1. I.Physikalisches Institut, Universität zu Köln, Zülpicher Str.77, 50937 Köln, Germany \\ 2. Max-Planck-Institut für Radioastronomie, Auf dem Hügel 69, 53121 Bonn, Germany
}

\begin{abstract}
We report on recent near-infrared (NIR) and X-ray observations of Sagittarius $\mathrm{A}^{*}$ (Sgr A*), the electromagnetic manifestation of the $\sim 4 \times 10^{6} \mathrm{M}_{\odot}$ super-massive black hole (SMBH) at the Galactic Center. The goal of these coordinated multi-wavelength observations is to investigate the variable emission from $\mathrm{Sgr} \mathrm{A}^{*}$ in order to obtain a better understanding of the underlying physical processes in the accretion flow/outflow. The observations have been carried out using the NACO adaptive optics (AO) instrument at the European Southern Observatory's Very Large Telescope (July 2005, May 2007, May 2008) in conjunction with X-ray and radio (sub-)mm observations using the Chandra X-ray observatory, CARMA ATCA

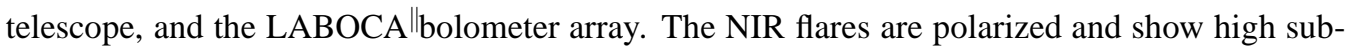
flare to flare contrast. The observations can be interpreted in the framework of a model involving a temporary disk with a short jet. The NIR variations may contain a significant contribution from an underlying physical process that can statistically be described as red-noise. In an accretion disk model they may be interpreted in the framework of a multi-component structure in a differentially rotating disk with occasionally dominated by a single hot-spot.
\end{abstract}

Identification of dark matter 2008

August 18-22, 2008

Stockholm, Sweden

\footnotetext{
${ }^{*}$ Speaker.

†E-mail: eckart@ph1.uni-koeln.de

¥Support for CARMA construction was derived from the states of California, Illinois, and Maryland, the Gordon and Betty Moore Foundation, the Kenneth T. and Eileen L. Norris Foundation, the Associates of the California Institute of Technology, and the National Science Foundation. Ongoing CARMA development and operations are supported by the National Science Foundation under a cooperative agreement, and by the CARMA partner universities.

$\S$ ATCA is operated by the Australia Telescope National Facility, a division of CSIRO, which also includes the ATNF Headquarters at Marsfield in Sydney, the Parkes Observatory and the Mopra Observatory near Coonabarabran.

IThe IRAM 30m millimeter telescope is operated by the Institute for Radioastronomy at millimeter wavelengths Granada, Spain, and Grenoble, France.

"The LABOCA bolometer array, located on the Atacama Pathfinder EXperiment (APEX) telescope. The Atacama Pathfinder Experiment (APEX) is a new-technology 12-m telescope, based on an ALMA (Atacama Large Millimeter Array) prototype antenna, and operating at the Llano Chajnantor at an altitude of $5105 \mathrm{~m}$. APEX is a collaboration between the Max-Planck-Institut für Radioastronomie, the Onsala Space Observatory and ESO.
} 


\section{Introduction}

The investigation of the dynamics of stars has demonstrated the existence of a super massive black hole (SMBH) at the center of the Milky Way. At a distance of only $\sim 8 \mathrm{kpc}$ a SMBH of mass $\sim 4 \times 10^{6} \mathrm{M}_{\odot}$ can convincingly be identified with the compact radio, infrared, and X-ray source Sagittarius A* (Sgr A*; Eckart \& Genzel 1996, Ghez et al. 1998, Eckart et al. 2002, see references in Eckart et al. 2008a). Additional strong evidence for a SMBH at the position of Sgr $\mathrm{A}^{*}$ comes from the observation of flare activity on hourly time scales both in the X-ray and NIR wavelength domain (Baganoff et al. 2003; Genzel et al. 2003).

Due to its proximity $\mathrm{Sgr} \mathrm{A}^{*}$ provides us with a unique opportunity to understand the physics and possibly the evolution of SMBHs at the nuclei of galaxies. Sgr A* is very compact (Doeleman et al. 2008) and remarkably faint ( $\leq 10^{-9}$ of the Eddington luminosity) in all wavebands. Its feeble emission is likely to be due to a combination of a low accretion rate with a low radiation efficiency. Mouawad et al. (2005) presented the first exemplary efforts to determine the amount of extended mass in the vicinity of the Black Hole $\mathrm{M}_{S M B H}=3-4 \times 10^{6} \mathrm{M}_{\odot}$ allowing for non-Keplerian orbits. Using positional and radial data of the star S2, the authors find that there could exist an unobserved extended mass component of several $10^{5} \mathrm{M}_{\odot}$ forming a so-called 'cusp' centered on the black hole position. Considering only the fraction of the cusp mass $\mathrm{M}_{S 2_{a p o}}$ within the apo-center of the S2 orbit, Mouawad et al. (2005) find as an upper limit that $\mathrm{M}_{S 2_{a p o}} /\left(\mathrm{M}_{S M B H}+\mathrm{M}_{S 2_{a p o}}\right) \leq 0.05$. A large extended cusp mass, if present, is unlikely to be composed of sub-solar mass constituents, but could be explained rather well by a cluster of high M/L stellar remnants, which form a stable configuration.

\section{Results from coordinated observations}

The temporal correlation between rapid variability of the near-infrared (NIR) and X-ray emission suggests that the radiation arises from a compact source within a few ten Schwarzschild radii of the SMBH (Eckart et al. 2004, 2006ab). For several simultaneous flare events the authors found no time lag larger than an upper limit of $\leq 10$ minutes, mainly constrained by the required binning width of the X-ray data. The flaring state can be explained with a synchrotron self-Compton (SSC) model involving up-scattered sub-millimeter photons from a compact source component. Inverse Compton scattering of the THz-peaked flare spectrum by the relativistic electrons then accounts for the X-ray emission. This model allows for NIR flux density contributions from both the synchrotron and SSC mechanisms. Observations for red and variable NIR flare spectra are indicative of a possible exponential cutoff of the NIR/MIR synchrotron spectrum (Eckart et al. 2006b, 2008a and references therein).

The NIR flare emission is polarized with a well limited range over which the position angle of the polarized emission is changing $\left(60^{\circ} \pm 20^{\circ}\right)$ (Eckart et al. 2006b, Meyer et al. 2006ab, 2007). The flux density variations of Sgr A* can be explained in a disk or jet model (see e.g. discussion in Eckart et al. 2006ab, 2008a), or they could be seen as a consequence of an underlying physical process that can mathematically be described as red-noise (Eckart et al. 2008a, Do et al. 2008). We find a light curve structure that consists of maxima separated by about 100 minutes with additional fluctuations of smaller amplitude. Assuming the presence of a disk and by simultaneous 
fitting of the previously obtained light curve fluctuations and the time-variable polarization angle, we have shown that the data can be successfully modeled with a simple relativistic hot spot/ring model (Meyer et al. 2006ab, 2007, Eckart et al. 2006ab, 2008b). In this model the broad nearinfrared flares ( $\sim 100$ minutes duration) of Sgr $\mathrm{A}^{*}$ are due to a sound wave that travels around the SMBH once. The sub-flares, superimposed on the broad flare, are thought to be due evolving (i.e. expanding) hot-spots that may be relativistically orbiting the central SMBH.

Inspection and modeling of the radio (sub-)mm and infrared light curves shows that the sub$\mathrm{mm}$ follows the NIR emission with a delay of $1.5 \pm 0.5$ hours (Eckart et al. 2006ab, Marrone et al.2008, Yusef-Zadeh et al. 2008). probably due to an adiabatic expansion of the source components. This is also supported by the results of two NIR/sub-mm runs: 1) The observations in May 2008 using NACO at the VLT and the LABOCA bolometer at the Atacama Pathfinder Experiment (APEX), at $0.87 \mathrm{~mm}$ wavelength (345 GHz) (Eckart et al. 2008c, García-Marín 2008 in prep.); 2) The coordinated observations in May 2007 using CARMA, ATCA, and the MAMBO bolometer at the IRAM 30m telescope (Kunneriath et al. 2008). The derived physical quantities that describe the flare emission give a source component expansion speed of $\mathrm{v}_{\exp } \sim 0.005 \mathrm{c}$, source sizes around one Schwarzschild radius with flux densities of a few Janskys and spectral indices of $\alpha=0.8$ to 1.8 , corresponding to particle spectral indices $\sim 2.6$ to 4.6. At the start of the flare the spectra of these components peak at frequencies of a few $\mathrm{THz}$. These parameters suggest that the adiabatically expanding source components either have a bulk motion larger than $\mathrm{v}_{\text {exp }}$ or the expanding material contributes to a corona or disk, confined to the immediate surroundings of Sgr A*.

\section{Alternatives to the black hole scenario?}

Explaining SgrA* with alternative solutions for a SMBH becomes increasingly difficult. Stellar orbits near SgrA* make a universal Fermion ball solution for compact galactic nuclei highly unlikely (see references in Eckart et al. 2008abc). The good agreement between the measured polarized flare structure and the theoretical predictions (e.g. Eckart et al. 2006b) as well as the indication of a quasi-periodicity in the data severely challenge the alternative explanation of the central mass as a massive boson star (Torres, Capozziello \& Lambiase 2000, Lu \& Torres 2003 and references therein). Such a massive boson star scenario could already be excluded for the nucleus of MCG-6-30-15 (Lu \& Torres 2003). In the case of a stationary boson star the orbital velocity close to the $\sim 3 \mathrm{R}_{S}$ radius LSO is already $\sim 3$ times lower than that of a Schwarzschild SMBH (Lu $\&$ Torres 2003) and relativistic effects are severely diminished and further reduced at even smaller radii. If the indicated quasi-periodicity is due to orbital motion then a stationary boson star can be excluded as an alternative solution for SgrA*, since in this case one expects the orbital periods to be larger.

\section{Summary and discussion}

Our coordinated observations of $\operatorname{SgrA}^{*}$ over the past years have shown that the flare spectra are peaked in the THz spectral domain with the result that bright NIR flares are synchronous to $\mathrm{X}$-ray flares and that the flaring source components expand adiabatically with a 1.5-2 hour delay between the $\mathrm{THz}$ and sub-mm domain. Polarized NIR sub-flare structure is in agreement with hot 
spot on orbiting the SMBH with relativistic speeds. A significant fraction of the overall varability may be described as red noise. Future observations at high angular resolution in the mm- and NIR wavelength domain will have to determine the disk and/or jet nature of the emission source associated with the SMBH at the position of SgrA*.

\section{References}

[1] Baganoff, F. K. et al., 2003, ApJ 591, 891

[2] Do, T.; Ghez, A.M.; Morris, M.R.; et al., 2008, arXiv0810.0446D, in press

[3] Doeleman, S.S., et al., 2008, Nature 455, 78D

[4] Eckart, A., \& Genzel, R., 1996, Nature 383, 415

[5] Eckart, A., Genzel, R., Ott, T., Schödel, R., 2002, MNRAS 331, 917

[6] Eckart, A., Baganoff, F. K., Morris, M., et al., 2004, A\&A 427, 1

[7] Eckart, A., Baganoff, F. K., et al., 2006a , A\&A 450, 535

[8] Eckart, A., Schödel, R., et al., 2006b, A\&A 455, 1

[9] Eckart, A., Baganoff, F. K., et al., 2008a, A\&A 479, 625

[10] Eckart, A., Schödel, R., Baganoff, F.K., Morris, M., et al. 2008b, JPhCS, in press

[11] Eckart, A., Schödel, R., García-Marín, M., Witzel, G., et al., 2008c, accepted by A\&A

[12] Genzel, R., Schödel, R., Ott, T., et al., 2003, Nature 425, 934

[13] Ghez, A. M., Klein, B. L., Morris, M., Becklin, E.E., 1998, ApJ 509, 678

[14] Kunneriath, D., et al. 2008, JPhCS, in press

[15] Lu, Youjun; Torres, Diego F., 2003, IJMPD 12, 63

[16] Mouawad, N., Eckart, A., Pfalzner, S.; Schödel, R., Moultaka, J.; Spurzem, R., 2005 AN 326, 83

[17] Marrone, D. P., Baganoff, F. K., Morris, M., et al., 2008, ApJ 682, 373

[18] Meyer, L., Eckart, A., Schödel, R., et al., Karas, V., 2006a, A\&A 460, 15

[19] Meyer, L., Schödel, R., Eckart, A., Karas, V., Dovciak, M., Duschl, W. J., 2006b, A\&A 458, L25

[20] Meyer, L., Schödel, R., Eckart, A., Duschl, W. J., Karas, V., Dovciak, M., 2007, A\&A 473, 707

[21] Torres, D.F., Capoziello, S., Lambiase, G., 2000, Phys. Rev. D 62, 104012

[22] Yusef-Zadeh, F., et al., 2008 ApJ 682, 361 